\title{
Educación Intercultural y TIC: claves pedagógicas de la innovación y el cambio social en el siglo XXI
}

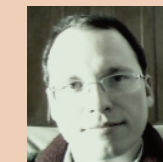

\section{Juan José Leiva Olivencia}

Profesor del Departamento de Didáctica y Organización Escolar de la Universidad de Málaga

juanleiva@uma.es

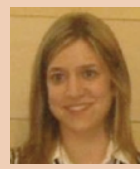

Diana Priegue Caamaño

Profesora del Departamento de Teoría de la Educación, Historia de la Educación y Pedagogía Social de la Universidad de Santiago de Compostela

diana.priegue@usc.es

|Fecha presentación: xx/xx/2012 | Aceptación: xx/xx/2012 |Publicación: 20/12/2012

\begin{abstract}
Resumen
Este artículo plantea la necesidad de ofrecer claves pedagógicas para la innovación y el cambio social en las instituciones universitarias y formativas del siglo XXI. Así, se pone de relieve la importancia de promover la educación intercultural a través de las TIC (Tecnologías de la Información y la Comunicación) como un elemento de innovación y de proyección positiva de la diversidad cultural. La cooperación en red desde una perspectiva intercultural y el impulso de las competencias interculturales son elementos fundamentales para transformar los escenarios formativos de una sociedad en permanente cambio social.
\end{abstract}

Palabras clave: sociedad del conocimiento, competencias interculturales, tecnología, digiculturalidad, comunidades de aprendizaje, aprendizaje cooperativo

\section{Resum}

Aquest article planteja la necessitat d'oferir claus pedagògiques per a la innovació i el canvi social a les institucions universitàries i formatives del segle XXI. Per tant, el que subratlla és la importància de promoure l'educació intercultural a través de les TIC (tecnologies de la informació i la comunicació) com a element d'innovació i projecció positiva de la diversitat cultural. La cooperació en xarxa des d'una perspectiva intercultural i la promoció d'habilitats interculturals són elements fonamentals per a transformar els escenaris formatius d'una societat en permanent canvi social.

Paraules clau: societat del coneixement, competències interculturals, tecnologia, digiculturalitat, comunitats d'aprenentatge, l'aprenentatge cooperatiu

\section{Abstract}

This article sets the need for teaching key for innovation and social change in universities and training institutions of the 21st century. So, it is highlighted the importance of promoting intercultural education through ICT (information and communications technologies) as an element of innovation and positive projection of cultural diversity. The networking from an intercultural perspective and the promotion of intercultural skills are fundamental elements to transform a training scenarios of a society in permanent social change. 


\section{Introducción}

Los continuos avances tecnológicos y el mayor protagonismo que han adquirido los flujos migratorios plantean nuevas exigencias, demandas y desafíos al ámbito pedagógico. Nos encontramos inmersos en una sociedad postmoderna y multicultural que aspira a la construcción de la interculturalidad en lo social y presencial, y a la digiculturalidad en lo cultural y virtual. Todo ello requiere que reflexionemos en mayor medida sobre el nuevo papel que, desde un paradigma inclusivo, comprensivo e intercultural, han de asumir las instituciones educativas aprovechando las posibilidades y oportunidades que nos ofrecen las TIC (Tecnologías de la Información y las Comunicaciones) para superar esa distancia entre la escuela y las realidades socioculturales. Atendiendo a lo expuesto por De Pablos (2009), al igual que el uso de las TIC en la enseñanza está teniendo un papel muy importante para lograr un replanteamiento metodológico, didáctico, curricular, organizativo en todos los niveles educativos con la mirada puesta en la optimización de los índices de calidad, también constituyen un instrumento valioso para aproximar culturas diferentes, reflexionar sobre la cultura de la diversidad, y promover una comunicación intercultural. Del mismo modo, para materializar los conceptos de interactividad, interconexión, instantaneidad, intercambio, conocimiento, enriquecimiento y ciudadanía intercultural, es necesaria una estrategia metodológica basada en el aprendizaje cooperativo, participativo y en red entre los centros de diversos lugares de nuestro mundo. Y para lograr este propósito las TIC constituyen una herramienta fundamental e imprescindible, no sólo por crear una comunidad donde los estudiantes pueden interactuar y compartir el conocimiento, sino por su potencial para transformar las prácticas educativas (Kozma y Anderson, 2002).

Es más, no hay duda que Internet y las redes sociales ponen a disposición de las escuelas inclusivas e interculturales todo lo necesario para generar y construir comunidades virtuales de aprendizaje intercultural, a través de multitud de herramientas susceptibles de favorecer la comunicación, la construcción compartida de conocimiento y el intercambio de información acerca de experiencias en educación intercultural entre todos los miembros de las comunidades educativas (De Haro, 2010).

2. De los conocimientos a las competencias: el potencial de las TIC en la sociedad del conocimiento A todas luces es claro que la incorporación de las tecnologías de la información y la comunicación (TIC) a los procesos de enseñanza-aprendizaje constituye uno de los mayores desafíos que debe afrontar nuestro sistema educativo. Nos referimos a un desafío que forma parte de un proceso de mayor calado, con repercusiones que afectan a las distintas dimensiones de nuestra sociedad, y tanto aquellos ámbitos que compartimos como ciudadanos como a muchos otros que nos incumben como individuos particulares.

En este contexto no debe sorprender la proliferación de distintos posicionamientos sobre el papel que tiene que desempeñar la tecnología en la sociedad de la información. Los planteamientos han sido variados, y aún contrapuestos, pero moviéndose entre dos extremos que van desde los discursos apocalípticos que insisten en el fin de los ideales y valores de modelo humanista de la cultura, hasta aquellos que hacen apología de los medios tecnológicos como la panacea de una sociedad más eficaz y llena de bienestar para sus ciudadanos (Area, 2009).
Como no podía ser de otra forma, las instituciones educativas también se han visto afectadas por las controversias derivadas de tan intensos debates. Esto se ha traducido en la difusión de varios enfoques (ver Mominó, Sigalés y Meneses, 2007), contrarios en determinados aspectos y complementarios en algunos otros, centrados en el estudio de la integración de las TIC en la educación.

En todo caso, en lo que sí hemos alcanzado un cierto consenso es en el reconocimiento de la influencia que ejercen los dispositivos tecnológicos en la vida de todos los ciudadanos pero, sobre todo, en la necesidad de asumir los desafíos educativos derivados de tal influencia. De acuerdo con Coll, Bustos y Engel (2007), la escuela debe revisar el viejo concepto de alfabetización y avanzar en la identificación de nuevas formas de alfabetización que faciliten el desarrollo de las competencias que demanda la sociedad actual. Sin lugar a dudas, disponer de las destrezas que implica el manejo de los medios tecnológicos, la capacidad para interactuar con personas de orígenes diversos, aprender a aprender, etc., son aspectos que hasta el momento han ocupado un segundo plano en los procesos de aprendizaje de nuestros estudiantes pero que hoy en día se han convertido en elementos imprescindibles en la formación de la ciudadanía del siglo XXI (Cummins y Sayers, 1995).

No es extraño, por tanto, que el concepto Lifelong Learning haya adquirido la consideración de pieza clave para el desarrollo económico y social de la Unión Europea. Si la formación de una persona no puede reducirse a una determinada etapa educativa, una de las prioridades de las instituciones educativas debe ser favorecer el desarrollo de las competencias necesarias para aprender de manera autónoma. Haciendo nuestras las palabras de De Miguel (2005: 17): el nuevo orden social "exige a cada sujeto una capacitación personal crítica que favorezca la interpretación de la información circulante y la generación del conocimiento propio que le permita aprender de forma continua".

$\mathrm{Y}$ es este aspecto donde las TIC nos abren nuevas posibilidades de acción educativa. Tal y como apuntan Borrero y Yuste (2011), la incorporación de las TIC en los ámbitos educativos, tanto formales como no formales, ofrecen múltiples y nuevas alternativas de acceso a la información y gestión del conocimiento. Hoy en día los canales de comunicación se multiplican vertiginosamente, la tecnología no para de innovar y el tiempo dedicado al consumo de los medios de comunicación crece a un ritmo acelerado. No obstante, y en total acuerdo con Francisco (2010), lo importante no es adquirir o asimilar información, sino integrarla y reconstruirla críticamente en el propio -y personal- conocimiento del saber, el saber en conocimiento y el conocimiento en cultura.

No podemos seguir sin detenernos en otro término de gran relevancia en la sociedad actual y que ha significado el inicio de una profunda transformación en las instituciones educativas de nuestro tiempo. Nos referimos al concepto de competencia que, como es bien sabido, a pesar de tener su origen en un contexto vinculado al empleo, en un periodo relativamente corto de tiempo, su alcance se ha dejado sentir con fuerza en las distintas reformas que se han llevado a cabo en los sistemas educativos de los países europeos. Concretamente, el proceso de reflexión en torno a las competencias en la educación básica, iniciado con el Informe DeSeCO (OCDE, 2005), culmina con la definición de un marco conceptual que pretende contribuir a la identificación de las denominadas competencias clave, es decir, el conjunto de 


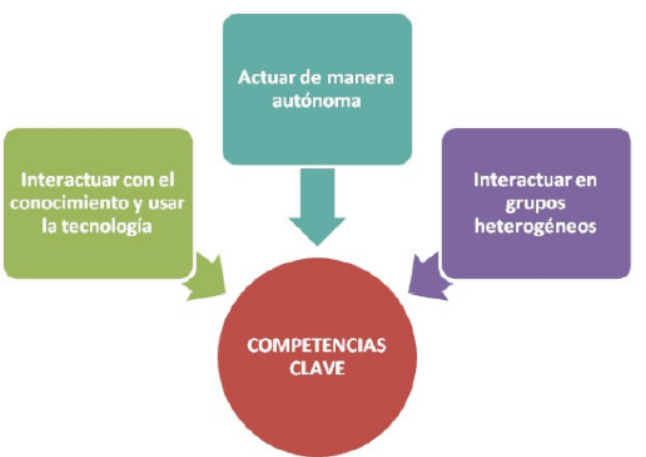

Figura 1: Bloques de competencias clave. Fuente: elaboración propia a partir de la propuesta de la OCDE (2005).

conocimientos, destrezas y actitudes que han adquirido la condición de imprescindibles en la sociedad contemporánea. La propuesta de la OCDE considera tres bloques de competencias clave (ver Figura 1), estrechamente vinculadas con varios términos que, como veremos, son fundamentales en nuestra propuesta.

Como no podía ser de otra forma, también en España se han producido cambios importantes en la normativa sobre educación, situándose entre los más relevantes las modificaciones introducidas en los currículos de los distintos niveles de enseñanza, tanto obligatorios como postobligatorios, con la aprobación de la Ley Orgánica 2/2006, de 3 de mayo, de Educación (LOE) (BOE 04/05/20011).

En clara consonancia con lo establecido en las directrices y recomendaciones internacionales, tanto en la LOE como en la normativa que la desarrolla, la educación se concibe como un aprendizaje permanente que debe desarrollarse a lo largo de la vida. Consecuentemente, nuestro sistema educativo debe garantizar a la ciudadanía la posibilidad de adquirir y ampliar los conocimientos, capacidades, actitudes y competencias necesarias para desarrollarse personal y profesionalmente tanto dentro como fuera de los contextos formales de aprendizaje.

La incorporación de las competencias básicas en los currículos de los niveles que componen la educación básica (educación primaria y educación secundaria obligatoria) responde a la necesidad de poner el acento en aquellos aprendizajes que se consideran imprescindibles para que los jóvenes puedan aprender a lo largo de su vida, realizarse personalmente, ejercer la ciudadanía activa, e incorporarse a la vida adulta de manera satisfactoria. Concretamente, y siempre tomando como referencia la propuesta realizada por la Unión Europea, se han identificado ocho competencias básicas: competencia en comunicación lingüística, competencia matemática, competencia en el conocimiento y la interacción con el mundo físico, tratamiento de la información y competencia digital, competencia social y ciudadana, competencia cultural y artística, competencia para aprender a aprender, y autonomía e iniciativa personal.

Tal y como se refiere en la citada ley, aunque las enseñanzas mínimas del currículo de la educación obligatoria son, a priori, suficiente garantía para que el alumnado alcance las competencias básicas, es preciso que tanto los distintos elementos que intervienen en el proceso de enseñanza-aprendizaje como la organización y funcionamiento de los centros promuevan su desarrollo.

Aunque este es un objetivo que incumbe prioritariamente a las etapas que componen la educación básica, la re- levancia de esta cuestión también requiere que los niveles postobligatorios contribuyan al perfeccionamiento y consolidación de las competencias básicas. A modo de ejemplo podríamos referirnos al Bachillerato. Tal y como se recoge en el Real Decreto 1467/2007, de 2 de noviembre, por el que se establece la estructura del bachillerato y se fijan sus enseñanzas mínimas (BOE 6/11/2007), entre los objetivos de las materias comunes de la etapa se encuentra profundizar en aquellas competencias que tienen un carácter más transversal y favorecen que los estudiantes continúen aprendiendo. De otra parte, las materias de modalidad deben promover el desarrollo de aquellas competencias que, además de estar más vinculadas con un determinado ámbito de conocimiento, preparan al alumnado para la realización de estudios posteriores y/o favorecen la inserción en un determinado campo laboral.

Si bien no profundizaremos en ello, también en la enseñanza universitaria hemos presenciado intensas reformas con la construcción del Espacio Europeo de Educación Superior (EEES) a partir de la Declaración de Bolonia que, entre otros aspectos, han motivado la incorporación de las competencias a los planes de estudio de las distintas titulaciones. En total acuerdo con Bolívar (2008), aunque la introducción de las competencias en los niveles previos a la universidad tiene un origen y finalidades distintas, ambos planteamientos coinciden en considerar que los conocimientos adquiridos tienen escasas posibilidades de transferencia fuera de los contextos formales de aprendizaje y, además, están lejos de resolver las demandas que la sociedad actual plantea a la ciudadanía.

Aunque resulta difícil establecer prioridades, y más aún cuando hablamos de educación, creemos que uno de los elementos que necesita ser profundamente revisado tiene que ver con los aspectos metodológicos. Tal y como insiste Vinagre (2010), la adquisición de las competencias que necesita el alumno exige poner el acento en los procesos de aprendizaje y no, como viene siendo habitual, en los procesos de enseñanza. En definitiva, de lo que se trata es de avanzar en la construcción de entornos que permitan a los estudiantes ser los auténticos promotores del proceso de aprendizaje, al tiempo que incrementan las posibilidades de interacción tanto con los iguales como con los propios docentes. No olvidemos que el conocimiento no es resultado de un proceso individual, es decir, no puede entenderse al margen del contexto en el que surge, sino dentro de un espacio social con el que el individuo interacciona.

Así es que, en un momento como el actual, caracterizado por una significativa ampliación de los escenarios de aprendizaje gracias a las diversas oportunidades de comunicación e interrelación que nos ofrecen los dispositivos tecnológicos, no debe extrañar la mayor preocupación social y cultural sobre el nivel de competencias que tienen los jóvenes en el uso de las TIC, una preocupación que se ha visto reflejada en la literatura científica-pedagógica de los últimos años (De Pablos, 2009; Monereo, 2009; Prendes, Castañeda y Gutiérrez, 2010) y ha traspasado los muros de la escuela para impregnar todos los ámbitos sociales y comunitarios. Y es este punto donde destacamos el potencial de la digiculturalidad como una competencia digital de conocimiento y valoración de la diversidad cultural a través de las TIC y con unas características complejas en su desarrollo metodológico y conceptual (Borrero y Yuste, 2011; Leiva, Yuste y Borrero, 2011; Priegue y Leiva, 2012). Como primera característica conceptual, subrayamos la necesidad de multialfabetizar a nuestro 


\begin{tabular}{|l|l|l|}
\hline Dimensión & Saber & Descripción \\
\hline Instrumental & Acceder a la información & $\begin{array}{l}\text { Adquirir las habilidades instrumentales para la } \\
\text { búsqueda de información y uso de las tecnologías }\end{array}$ \\
\hline Cognitiva & $\begin{array}{l}\text { Transformar la información en } \\
\text { conocimiento }\end{array}$ & $\begin{array}{l}\text { Saber plantear problemas, analizar e interpretar con } \\
\text { significado la información }\end{array}$ \\
\hline Sociocomunicacional & Expresarse y comunicarse & $\begin{array}{l}\text { Disponer de las habilidades para crear } \\
\text { documentos, difundirlos e interaccionar socialmente }\end{array}$ \\
\hline Axiológica & $\begin{array}{l}\text { Usar democrática y éticamente la } \\
\text { información }\end{array}$ & Desarrollo de actitudes, valores y prácticas sociales éticas \\
\hline
\end{tabular}

Tabla 1. Dimensiones de aprendizaje (Area, 2010).

alumnado para que sepa adaptarse a los diferentes cambios y necesidades de la sociedad de hoy. En este sentido, Area (2010), citado en Borrero y Yuste (2011) destaca las dimensiones o ámbitos de aprendizaje de la alfabetización tecnológica, que resumimos en la Tabla 1.

La segunda característica de la digiculturalidad corresponde a su desarrollo y aplicabilidad práctica en red. Esto significa que fundamentalmente tiene sentido pedagógico en tanto se genera y construye a partir de los valores de cooperación e interacción cultural democrática. En efecto, la digiculturalidad emerge con fuerza a través de plataformas y comunidades virtuales que son a la vez camino y destino de una conciencia comunitaria e intercultural donde lo importante es crecer individual y socialmente valorando positivamente la diversidad cultural y los valores universalmente aceptados contemplados en los derechos humanos (Martínez, 2010). Así mismo, no queremos terminar este apartado sin exponer qué es lo que las TIC están aportando al mundo de la interculturalidad. En este sentido nos gustaría destacar cuatro características básicas que servirán de soporte para el siguiente punto. Así, está claro que a través de las TIC podemos:

- Motivar a los más jóvenes a participar en la generación de comunidades virtuales.

- Promover el conocimiento y reconocimiento de las diferencias culturales como claves positivas de enriquecimiento personal y social.

- Facilitar la comunicación intercultural a través del contacto de personas y culturas muy diversas.

- Favorecer la participación activa y colaborativa gracias al empleo sistemático de herramientas virtuales de la Web 2.0.

Por tanto, el desafío que plantea la generación de todos los niveles educativos, especialmente en aquel alumnado universitario de las titulaciones conducentes a ser profesionales de la educación sería adquirir competencias interculturales desde un nuevo enfoque de formación universitaria, más preocupada por los principios de procedimiento didáctico-estratégicos y los valores educativos de la diversidad y el respeto cultural, que por meras aplicaciones o conocimientos teóricos de una aparente y aséptica tecnología educativa. Esto, de acuerdo con lo planteado por Valls y Otros (2002), supone que el profesorado universitario comience a incentivar y a generar en las aulas universitarias procesos didácticos en términos de comunidades de aprendizaje, lo cual exige la puesta en marcha de todo un conjunto de iniciativas que, desde la transformación de la organización escolar y de su funcionamiento, culmine en la acogida y promoción de la diversidad cultural como un eje formativo fundamental en el aprendizaje de las competencias digiculturales. A examinar esta cuestión dedicaremos las páginas siguientes.

\section{La cooperación en la red desde los parámetros de la interculturalidad}

Cierto es que una de las principales funciones de cualquier sistema educativo es posibilitar el desarrollo integral del alumnado. Esta tarea nunca ha sido fácil, pero podemos afirmar que hoy día se ha convertido en un reto aún mayor debido a los profundos y acelerados cambios sociales que se vienen produciendo (Esteve, 2004). Uno de los factores fundamentales de dichos cambios ha venido motivado por una creciente multiculturalidad en nuestras sociedades, que si bien nunca han sido homogéneas, dado que la diversidad cultural es algo consustancial a la propia sociedad, sí es cierto que en el caso español y en poco más de dos décadas esta presencia de personas de culturas diversas ha crecido exponencialmente, configurando una sociedad en la que se ha hecho más que evidente la pluralidad de identidades culturales.

El fenómeno de las relaciones interculturales se ha convertido en un tema de máximo interés para gran número de profesionales dedicados a distintos campos de estudio. Por su parte, la educación intercultural ha adquirido la consideración de herramienta fundamental de cara a superar los prejuicios que existen hacia los grupos minoritarios, así como para conseguir una interacción cultural en el contexto educativo basada en el mutuo enriquecimiento (Merino y Ruiz, 2005). Es por ello que cuando hablamos de educación intercultural estamos planteando estrategias de innovación con la finalidad de producir cambios en los procesos educativos para que éstos se adapten tanto a las necesidades del alumnado como a las exigencias sociales del momento.

En este sentido es importante poner de relieve el hecho de que la educación intercultural debe tener presente a todos los escolares y no únicamente a aquellos alumnos pertenecientes a minorías, es decir, "este tipo de educación va encaminada a conseguir en todos los alumnos, de todos los centros, a través de cualquier área y ámbito curricular, una sólida competencia cultural; es decir, una serie de aptitudes y actitudes que capaciten a todos los alumnos para funcionar adecuadamente en nuestras sociedades multiculturales y multilingües" (Jordán, 1996: 27-28).

La escuela es un lugar privilegiado para la interculturalidad, ya que frente a las asimetrías que se dan en las interacciones en la mayoría de los ámbitos sociales, en el centro escolar podemos ejercer un cierto control sobre cómo se producen esas interacciones, donde podemos generar relaciones, marcos de participación, intercambios y prácticas que puedan desarrollarse en condiciones de razonable igualdad (Lluch y Salinas, 1997). Ahora bien, cabría cuestionarse si el curriculum que se desarrolla en nuestras escuelas es un curriculum justo e igualitario. La realidad parece indicar lo contrario, ya que el estilo de trabajo escolar, en muchas ocasiones, no se corresponde con el deseable para el fomento 
de una educación pluralista, en la que el desarrollo de actitudes y valores y el diálogo constante, abierto y argumentado debería ganar terreno frente a una obsesión evaluadora del rendimiento escolar, en el que los alumnos de cultura minoritaria se encuentran en clara desigualdad de condiciones. Y es precisamente por esta causa por la que deseamos plantear una serie de ejes básicos con el objetivo de ayudar en la verdadera construcción de una escuela inclusiva en la que todos y todas tengan cabida, puedan participar en igualdad de condiciones y con la que se sientan identificados, ya que la presencia de la educación intercultural en la escuela no se limitará en ningún caso a una mera inclusión de contenidos referidos a las distintas culturas de los alumnos (algo que también habrá que realizar), sino a un proceso integral de análisis y reflexión, a partir del cual se impliquen todos los elementos de la comunidad (Merino, Leiva y Pedrero, 2012).

El discurso y las prácticas tendentes a la integración han sido de un valor inestimable de cara al tratamiento de la educación intercultural en contextos educativos. Ha supuesto superar visiones reduccionistas, que implicaban que la mera presencia de alumnos de distintas culturas en un mismo contexto suponía ya de por sí intercambio y enriquecimiento cultural. A pesar de ello, con la entrada en el debate y en las prácticas educativas de la idea de "escuela inclusiva" se ha ido un paso más allá; en este sentido "la educación inclusiva supone dos procesos interrelacionados: el proceso de incrementar la participación de los alumnos en la cultura y el currículum de las comunidades y escuelas ordinarias, y el proceso de reducir la exclusión de los alumnos de las comunidades y culturas normales" (Parrilla, 2002: 18).

En lo que respecta al tratamiento de la educación intercultural en nuestro sistema educativo podemos decir que hemos pasado de un periodo de exclusión clara de aquellos sujetos que no pertenecían a lo que podríamos denominar como cultura mayoritaria (fundamentalmente población de etnia gitana) hasta llegar en la actualidad a enfoques interculturales que responden a principios de escuela inclusiva. En este largo camino hemos pasado por la escolarización segregada de estos alumnos en las mal denominadas escuelas puente para alumnos gitanos y por un enfoque compensador que, de una u otra manera, asimilaba diferencia cultural con déficit. Estamos de acuerdo con $\mathrm{Me}-$ rino cuando afirma:

"la expresión escuela inclusiva adquiere cada vez más aceptación como expresión adecuada para reflejar el modelo de escuela centrado en la comunidad que posibilita a cada individuo desarrollarse en igualdad de condiciones como persona y como ciudadano. La escuela inclusiva es una escuela equitativa y no discriminatoria en el sentido de exclusora o marginadora, una escuela que promueve y desarrolla procesos educativo-sociales equitativos, y que ayudan al hombre a construir y desarrollar su personal proyecto de vida individual y ciudadano" (Merino, 2009: 49).

Por tanto, plantear los procesos educativos interculturales como ejes centrales de innovación en la escuela inclusiva supone apostar, por una parte, por la presencia en la vida del centro de toda una serie de valores y actitudes prosociales que vayan encaminadas a la consecución de una igualdad real y efectiva de todos las personas que forman parte de la comunidad y, por otra parte, por una apertura de esta comunidad educativa hacia el entorno, hacia la socie- dad, en la que los distintos agentes se sientan representados y partícipes de este proyecto. Para ello será necesaria una redefinición de la propia escuela y de sus metodologías, con el fin de que respondan a las nuevas realidades sociales de las que venimos hablando (Merino, 2009).

Es por ello que en este trabajo apuntamos algunos ejes que pueden ir en esta dirección, tratando de "articular respuestas educativas capaces de hacer frente a las demandas de flexibilidad, innovación, apertura y cooperación para poder atender a la diversidad del alumnado en condiciones de igualdad y sin recurrir a la exclusión" (Pujolás, 2010: 38). Efectivamente la lucha contra la exclusión debe ser una constante en la labor educativa de este tipo de escuela por la que apostamos, y esta tarea en la actualidad, en un contexto de crisis económica aguda, demanda que se articulen más que nunca todos los medios necesarios para favorecer la igualdad y la inclusión. A este respecto, Parrilla (2002) expone que la exclusión no es una situación reducida al siglo XX, es bien cierto que las exclusiones son hoy mayores. Ocupan de hecho un lugar importante en los discursos científicos, sociales y políticos. El efecto excluyente de la globalización y los grandes cambios de la nueva era han supuesto la aparición cada vez mayor de personas y regiones enteras que viven al margen de la sociedad, y ponen de manifiesto la necesidad primera de luchar contra la exclusión social.

La educación intercultural abordada desde un enfoque inclusivo supone la participación de todos los alumnos y de la comunidad en la vida escolar, valorando las diferencias, evidenciando aquello que nos es común y respetando al individuo:

"El punto de partida de la inclusión es la igualdad inherente a todas las personas, y de ahí la igualdad de derechos humanos que da pie a todo el desarrollo del movimiento inclusivo. La inclusión no habla, o no habla sólo del derecho de determinadas personas a vivir y gozar de unas condiciones de vida similares a las del resto de los ciudadanos, sino del derecho y la obligación social de construir entre todos comunidades para todos, comunidades que permiten valoran la diferencia, pero basadas en el reconocimiento básico y primero de la igualdad" (Parrilla, 2002: 25).

Esto, como ya hemos comentando, implica cambios profundos en la estructura escolar, y por tanto retos a los que tiene que hacer frente una institución que tradicionalmente se ha caracterizado por su inmovilismo. El profesor Merino Fernández (2009) expone dos desafíos o retos a los que la escuela actual se enfrenta en su esfuerzo de innovación y replanteamiento para seguir siendo útil a la educación de los hombres y contribuir al desarrollo de la sociedad:

a) El reto cultural y científico-profesional que adquiere características diferentes a las tradicionales debido a la aceleración, producción y caducidad del conocimiento y de la información.

b) El reto vital-convivencial desencadenado por las dificultades para la convivencia que genera la diversidad y el acercamiento e interacción inmediata (virtual o in situ) de individuos y comunidades humanas.

No olvidemos, además, tal y como plantea AbdallahPretceille (2001: 41), que "el discurso intercultural se centra en el mundo de las interacciones, partiendo del supuesto o 
concepción de la persona como ser comunicativo". Es por ello que el eje de interés de la educación intercultural no debe ser otro que la promoción de las condiciones educativas deseables para que personas de diversas culturas se encuentren, convivan, y vayan tejiendo entre ellas lazos de verdadera comunicación; de una comunicación interpersonal en la que primen genuinas actitudes de respeto, empatía, solidaridad, valoración y responsabilidad recíproca.

$Y$ es en este punto donde nos encontramos con un concepto clave en el enfoque intercultural y cuyo desarrollo cobra sentido en los procesos de interacción social. Nos referimos a la comunicación intercultural que, siguiendo a Vilà (2005), puede ser definida como la comunicación interpersonal en la que intervienen sujetos con referentes culturales distintos y con manifiestas diferencias, lo cual exige superar algunas barreras personales y/o contextuales para llegar a comunicarse de forma efectiva.

En estrecha relación con esta cuestión debemos tener presente que lo realmente relevante de las TIC no es su potencial para facilitar el acceso a la información, sino sus posibilidades para incrementar los procesos de comunicación interpersonal, al margen de las dimensiones espacio-tiempo y la construcción compartida del conocimiento. Es lo que Tubela y Vilaseca (2005) definen como modelo de ciberespacio de comunidad, cuyo eje central es la comunicación entre usuarios, el establecimiento de vínculos entre los participantes y la gestión democrática del conocimiento que se construye en la comunidad. Así pues, las comunidades virtuales de aprendizaje se caracterizan porque, además de constituirse como una comunidad de intereses o de participación, utilizan los soportes digitales como infraestructura para consolidar y ampliar las redes de comunicación y de intercambio, al tiempo que como instrumento para promover el aprendizaje de sus miembros (Coll, Bustos y Engel, 2007). Claro está que si la educación es un proceso de comunicación, cuando hablamos de interculturalidad esta afirmación cobra aún mayor firmeza, por ser este el ámbito donde el sujeto de la educación no puede entenderse aisladamente (Santos, Lorenzo y Priegue, 2011).

Asimismo, no cabe duda de que el E-Learning en el contexto educativo está transformando en los últimos años el proceso de enseñanza y aprendizaje, y los roles de docentes y alumnado cambian favoreciendo un enfoque didáctico centrado en el alumno que mejora la autonomía respecto de su propio aprendizaje (Cabero, López y Llorente, 2009). Se hace difícil hoy en día pensar en centros educativos de calidad y excelencia que funcionen sin el soporte de las TIC, ya que gran parte de la docencia que ésta realiza se sustenta en las tecnologías (Aguaded y Pérez, 2007). No obstante, todavía queda un largo camino que recorrer para que las escuelas interculturales incorporen a sus acciones y diseños pedagógicos elementos tecnológicos que animen y promuevan la participación digital desde un enfoque intercultural.

Las escuelas interculturales deben cambiar, y la gran mayoría así lo está haciendo, centrando los procesos de formación didáctica en las características y necesidades de los estudiantes, e incorporando escenarios flexibles, dinámicos, versátiles y abiertos para la formación y el aprendizaje basado en competencias de aprendizaje relevantes (López, 2009). Además, cabe señalar aquí que los niños y jóvenes de hoy son cada vez más nativos digitales y las aulas deben afrontar el reto de dar clase a jóvenes socializados ya en un mundo virtual cada vez más emergente (Prensky, 2001). Igualmente, no podemos negar la necesidad de vincular de manera ineludible TIC, innovación educativa y educación intercultural en escuelas donde el aprovechamiento educativo de la diversidad cultural se puede hacer con relatividad facilidad si existe voluntad pedagógica para ello. Y esto es así en la medida en que los centros e instituciones escolares del siglo XXI ya están empezando a emplear masivamente las TIC y las redes sociales como elementos de difusión y transformación de la información y el conocimiento. Igualmente, decir que TIC va unido a la mejora de los procesos de modernización, mejora e innovación en la docencia universitaria es algo que hoy en día no asombra a nadie. Ahora bien, las instituciones escolares deben promover la educación para el talento, y como tal, la utilización de las herramientas virtuales de aprendizaje es un elemento de gran potencialidad pedagógica y comunicativa para alumnado y docentes. La innovación educativa en el contexto educativo multicultural no radica solamente en la valoración presencial de la interculturalidad, también su impulso a través de las TIC y aprovechando las plataformas y webs de los centros educativos, puede ser un motivo conductor de enorme potencialidad para que las familias, tanto autóctonas como inmigrantes, sean proactivas en la generación de lo que venimos denominando digiculturalidad. Por este motivo, la innovación educativa intercultural debe subyacer en los procesos proactivos y eficientes que se pueden generar con las TIC y en los resultados de aprendizaje que se pueden obtener con una amplia variedad y diversidad de actividades de enseñanza y aprendizaje, no sólo para el alumnado, sino globalmente para todos los agentes de la comunidad educativa.

Aunque las ventajas de las TIC en los entornos educativos han sido objeto de exploración en los últimos años, encontramos que la tendencia predominante es su consideración como artefactos que contribuyen a la mejora de los procesos de enseñanza-aprendizaje, prevaleciendo claramente una visión excesivamente instrumental y de apoyo. Desde nuestro punto de vista, los beneficios de los dispositivos digitales han sido, comparativamente, menos examinados, lo cual no deja de ser un hecho sorprendente, dadas las numerosas referencias bibliográficas que insisten en la necesidad de una mejor capacitación profesional y en la urgencia de disponer de una preparación acorde a los nuevos desafíos de la sociedad actual, entre los que también se sitúa la mejor gestión de la diversidad étnico-cultural.

Es por ello que debemos tener en cuenta las numerosas evidencias empíricas que han constatado cómo el trabajo entre pares o expertos, es decir, los procesos de interacción en los procesos de enseñanza-aprendizaje, incrementan las posibilidades de éxito (Rogoff, 1993). En no pocas ocasiones a este tipo de trabajo se le ha llamado cooperativo o colaborativo, llegando a utilizarse ambos términos en la bibliografía científica como sinónimos, pero también como antónimos. Pues bien, aunque no nos detendremos en explicar las diferencias entre ambos métodos (ver Barkley, Cross y Mayor, 2007), antes de seguir, queremos dejar claro que nuestra propuesta se fundamenta en estructuras de interacción cooperativa, cuyo afán es más bien el desarrollo de la reflexión y la autonomía, en la génesis y desarrollo de la atracción interpersonal.

Naturalmente, de las potenciales virtualidades que presentan las técnicas de aprendizaje cooperativo nos interesa especialmente la mejora que parecen producir en el esfuerzo por el aprendizaje entre compañeros, porque aumentan la motivación general, la implicación en las tareas y la ayuda que se proporcionan entre sí, amén de que propicia la re- 
ducción de prejuicios étnicos. Las investigaciones realizadas sobre el aprendizaje cooperativo en equipos heterogéneos apoyan dicha teoría, así como la eficacia de la cooperación para favorecer la tolerancia y la integración de todos los alumnos en contextos interétnicos (Slavin, 1999; DíazAguado, 2003).

Los espacios en los que se coopera crean comunidades de ayuda en el estudio y en la indagación, en las cuales los alumnos se ven a sí mismos con responsabilidades específicas en los planos individual y grupal, independientemente de etnia, edad, clase o género. Repárese en la excelente oportunidad formativa que ofrecen aquellas situaciones escolares que, por su composición heterogénea, permiten un fructífero conocimiento de las distintas señas de identidad que caracterizan a los miembros de la comunidad educativa. En idéntica perspectiva se sitúan Aguado, Gil y Mata (2008) cuando afirman que la formación en redes constituye en sí misma una experiencia intercultural de colaboración, que redunda en enriquecimiento y desarrollo integral.

Seguidamente se recogen las etapas que, a nuestro parecer, conformarían la creación de una comunidad virtual de aprendizaje. Para ello hemos realizado una adaptación de la propuesta de Salmon (2000; citado en Martín y Quiroz, 2006: 312):

- $1^{\text {a }}$ etapa: Acceso y motivación. El principal objetivo es reconocer la utilidad práctica y efectiva que una comunidad virtual de aprendizaje puede proporcionar al grupo en general y a cada individuo particularmente. Claro está que, previamente, es necesario contar con determinadas competencias tecnológicas, caso del manejo del hardware y software implicado en el acceso a las plataformas virtuales.

- $2^{\mathrm{a}}$ etapa: Compromiso y socialización en línea. Se trata, principalmente, de establecer identidades virtuales y relaciones entre los participantes. La calidad de los vínculos establecidos determinará en gran medida el desarrollo de las etapas posteriores.

- $3^{\text {a }}$ etapa: Intercambio de información. La finalidad es conseguir compartir información relevante en función de las propias necesidades, las de los otros participantes y de la comunidad en su totalidad. Además, es importante que los implicados perciban los aspectos positivos derivados del proceso.

- $4^{\mathrm{a}}$ etapa: Construcción del conocimiento. Ésta es una de las fases clave, pues el objetivo es lograr la construcción co-participativa de nuevos conocimientos a partir de las aportaciones de todos los participantes.

- $5^{\text {a }}$ etapa: Desarrollo. Se trata de usar el proceso para alcanzar metas personales, e integrar los conocimientos derivados de la implicación en la red con lo aprendido en otros contextos.

- $6^{\text {a }}$ Consolidación. El propósito de esta última etapa es que la red permanezca en el tiempo, o sea el origen de nuevas redes con objetivos más amplios. Para que podamos hablar de consolidación, el nivel de implicación de los participantes debe mantenerse.

A modo de síntesis, podríamos decir que las claves que favorecen el éxito de este tipo de comunidades pasan por compartir objetivos o intereses comunes, tener un sentimiento de pertenencia al grupo, y un compromiso activo de todos los miembros. Por otra parte, tampoco debemos pasar por alto que los estudios realizados a nivel internacional han demostrado que la participación del alumnado en estas estructuras incide positivamente en el rendimiento académico y en la motivación de los estudiantes (Thomas, 2000). A todo ello debemos añadir su gran potencial para la creación de espacios de interacción cooperativa. Naturalmente, de las potenciales virtualidades que presentan las técnicas de aprendizaje cooperativo nos interesa especialmente la mejora que parecen producir en el esfuerzo por el aprendizaje entre compañeros, porque aumentan la motivación general, la implicación en las tareas y la ayuda que se proporcionan entre sí, amén de que propicia la reducción de prejuicios étnicos (Slavin, 1999; Santos, Lorenzo y Priegue, 2009). Concretamente, el potencial de estos soportes en el desarrollo de competencias de corte intercultural tiene que ver con cuatro aspectos fundamentales en los que nos centraremos a continuación.

En primer lugar, todos los participantes aportan su actividad a fin de alcanzar una meta común, siendo ésta otra de las premisas básicas del aprendizaje cooperativo. Pensemos en la idoneidad de estas estructuras para la asunción de responsabilidades compartidas y el establecimiento de metas de equipo, por encima de comportamientos individualistas y de la competitividad, relegando a un segundo plano las diferencias que puedan existir entre los participantes y fortaleciendo el sentimiento de comunidad y necesidades compartidas. Así pues, una de las claves del éxito reside en crear interdependencia positiva entre los integrantes del grupo, es decir, la necesidad de que trabajen juntos para conseguir los objetivos establecidos en y para la comunidad.

Otro de sus aspectos positivos tiene que ver con su contribución a la educación en y para la democracia. La naturaleza del uso y funcionamiento de estos soportes favorece el establecimiento de estructuras de participación democráticas, donde se combinan aprendizajes de tipo cognitivo, social y emocional. El diálogo, la confrontación de ideas y la participación son herramientas esenciales para el buen funcionamiento de estas estructuras y, al mismo tiempo, todos ellos son elementos fundamentales para el desarrollo de una sociedad democrática (Santos y Lorenzo, 2009).

El tercer aspecto a destacar es que la desaparición del espacio, como distancia física entre los interlocutores, no lleva emparejada la desaparición del entorno social que rodea a esos mismos interlocutores (Martínez y Prendes, 2003). Es evidente que los procesos de comunicación se establecen y se construyen desde los espacios de significación cultural de cada individuo, por lo cual la implicación en redes de este tipo posibilita el conocimiento de realidades y representaciones culturales diferentes, lo que es susceptible de contribuir a la crítica de las propias identidades culturales (individuales y colectivas), así como a la generación de una cultura de la diversidad en el espacio virtual.

En último lugar, la participación en estas redes favorece la aceptación y la valoración positiva de la diversidad. No nos referimos únicamente a las diferencias que puedan existir por razones culturales o de género, sino a la diversidad intrínseca que nos es propia como seres particulares.

Una vez expuestos los aspectos fundamentales de nuestra propuesta, hemos de resaltar que existen varios entornos virtuales que hacen posible su puesta en práctica en las aulas de todos los niveles educativos. Además de la plataforma Moodle, disponemos de otros sistemas menos conocidos, caso del BSCW (Basic Support for Cooperative Working) que, como su propio nombre indica, constituye un entorno basado en el trabajo cooperativo en la red que, además de la 
navegación y el acceso a la información, permite compartir y construir documentos a través de distintas plataformas (Windows, Macintosh o Unix) y establecer una agenda común entre los miembros del grupo. Dispone, además, de distintas herramientas para supervisar la creación, lectura o modificación de información a través de un sistema de alerta que nos informa de la actividad de otros usuarios del entorno. Todo ello sin que sea necesario instalar ningún software adicional. Aunque en este trabajo tan sólo nos refiramos a una de ellas, en los últimos años se han desarrollado numerosas herramientas groupware bajo licencias de software libre de fácil acceso, con prestaciones muy similares a las que nos proporcionan distintas marcas comerciales, habitualmente menos accesibles por su elevado coste. Ahora bien, sabiendo que disponemos de los soportes tecnológicos necesarios para desarrollar un planteamiento como el que desde aquí proponemos, probablemente su rentabilización desde un punto de vista intercultural dependerá en gran medida de la formación y actitudes no sólo de los docentes, sino también de la comunidad escolar en su conjunto, además de la formación intercultural del profesorado en iniciativas y diseños didácticos especializados en el empleo reflexivo y crítico de las TIC para la promoción de las relaciones interculturales entre los diferentes agentes educativos (Luchtenberg, 1995; Tondeur et al., 2009).

\section{Experiencias de Educación Intercultural a través de las TIC}

En las aulas, en mayor o menor grado, y gracias al buen quehacer profesional de los docentes, se han ido integrando las TIC en sus programaciones y procesos de enseñanza-aprendizaje acordes con su aparición y contexto (televisión, vídeo, retroproyectores, Internet, redes sociales, etc.). Además, al margen de las carencias que el profesorado percibe en su formación para el manejo y aprovechamiento de las TIC, los estudios dan cuenta de la mayor sensibilidad que ha ido desarrollando este colectivo hacia la necesidad de introducir las tecnologías en el proceso de enseñanza-aprendizaje, sobre todo, por su gran potencial para diversificar el proceso de enseñanza-aprendizaje y de atender, en mayor medida, las necesidades particulares que pueda presentar el alumnado, especialmente el procedente de la inmigración.

No podemos continuar sin reconocer el notable esfuerzo económico que ha realizado la administración educativa para proporcionar a los centros escolares el equipamiento tecnológico que exige una sociedad que apuesta por la mayor rentabilización del espacio digital. A este nivel, uno de los referentes en nuestro país lo encontramos en la Comunidad Autónoma de Extremadura, donde en el año 1999 se presentaba el Plan Estratégico para el Desarrollo de la Sociedad de la Información, siendo el sector educativo uno de los pilares sobre los que se asentaba dicho desarrollo. Es a partir de este momento cuando en Extremadura aparece lo que se ha denominado Las TIC en el Aula (Borrero y Yuste, 2011). En este contexto nace LinEx que no sólo es un sistema operativo, sino que actualmente posee un conjunto enorme de importantes y variadas aplicaciones informáticas que cubre las necesidades de usuarios básicos y avanzados, al tiempo que ofrece diversidad de herramientas para uso en el aula. A partir del año 2000, se inicia el despliegue de la Red Tecnológica Educativa, que se completa en el año 2003. Las TIC se hacen presentes en el aula, no son ya un recurso externo sino una herramienta más, como la tiza o la pizarra. Todos los centros educativos públicos, disponen de conectividad de banda ancha con un parque informático de unos 70.000 ordenadores, lo que posibilita una ratio de un ordenador por cada dos alumnos en secundaria y uno por cada seis en primaria: aparece el concepto de Aula Tecnológica. Ahora bien, la educación intercultural precisa de objetivos más amplios: reclama instituciones y personas que necesariamente trabajen en red, en comunidades de aprendizaje que promuevan la diversidad cultural como una riqueza, y no una lacra perturbadora de la convivencia o del rendimiento académico, lo cual supone todo un conjunto de iniciativas que deberán culminar con la transformación de la organización escolar. En efecto, lo que necesitamos es impulsar procesos de comunicación e intercambio intercultural intra e intercentros fundamentados en el reconocimiento del potencial educativo de la diversidad y de la acción comunitaria, empleando nuevos entornos virtuales de comunicación e interacción.

De hecho, desde el Observatorio Escuela 2.o, del Ministerio de Educación, se plantea que ante el fenómeno de la inmigración y la diversidad cultural, las instituciones escolares deben tener presente en su gestión que en ellas se encuentra alumnado procedente de diferentes culturas y con diferentes lenguas, que tienen que integrarse en un marco de convivencia común y experimentar la diversidad no como un obstáculo, sino como un fenómeno positivo; comprometerse con el principio de igualdad de oportunidades en la educación y la convivencia y vertebrarse desde un enfoque comunitario del hecho educativo y de la construcción de una nueva ciudadanía, necesariamente intercultural. Estamos ante un proceso que debe ser liderado por las instituciones educativas y sus profesionales. Sin embargo, al igual que ocurre con otras cuestiones de gran relevancia pedagógica, la escuela y los docentes no pueden asumir en solitario la mejor formación del alumnado, sobre todo, cuando sabemos que lo que precisamos es impulsar procesos formativos fundamentados en los principios de la interculturalidad y, en consecuencia, debemos contar con el compromiso de las demás agencias educadoras. En este sentido, resulta muy relevante destacar el trabajo realizado en la Comunidad Autónoma de Andalucía, donde la Consejería de Educación viene impulsando el desarrollo de nuevas estrategias educativas de cooperación, formación y participación de las familias. De hecho, la Consejería de Educación, a través de la Plataforma Educativa Helvia, está facilitando la creación de redes de innovación e investigación, la base andaluza de recursos digitales (BARTIC), un banco de materiales digitales accesible a toda la ciudadanía donde se podrán buscar y descargar juegos, experiencias educativas, contenidos escolares y objetos de aprendizaje, así como espacios virtuales destinados a las comunidades educativas para facilitar la posibilidad a docentes, familias y alumnado de crear sus propias weblogs, foros y bitácoras en los que intercambiar reflexiones, ideas, propuestas y recursos educativos diversos.

De similar envergadura es el esfuerzo realizado en la Comunidad Autónoma de Galicia para poner en marcha desde el curso 2010-11 el Proyecto Abalar, una iniciativa que tiene por objetivo potenciar la integración plena de las TIC en la práctica educativa en esta Comunidad y que apuesta por el concepto de centro educativo digital. Por y para ello, desde la Consellería de Educación e Ordenación Universitaria de la Xunta de Galicia se ha realizado una gran inversión económica, a fin de dotar a los centros escolares de los recursos humanos y pedagógicos, así como de las infraestructuras y servicios necesarios, para el aprovechamiento cotidiano de 
la tecnología por parte de todos los agentes de la comunidad educativa. Además de promover distintas acciones formativas para mejorar la preparación del profesorado en el manejo y aprovechamiento de los soportes tecnológicos, en el marco del proyecto se ha creado un portal digital (Espazo Abalar) que aloja recursos educativos en soporte digital para los distintos niveles de enseñanza que los docentes pueden utilizar en sus materias y dispone de varias aplicaciones telemáticas que facilitan la comunicación entre los distintos miembros de la comunidad educativa. Sin dejar de reconocer los avances que pueden derivarse de la puesta en marcha de soportes digitales que promueven la colaboración entre la escuela y los demás agentes educativos, probablemente el aprovechamiento de sus ventajas desde una perspectiva intercultural dependerá tanto de la buena voluntad de los docentes como de la formación que hayan recibido para ello. En este sentido, uno de los proyectos donde se puede observar con nitidez la vinculación y traducción práctica de la interculturalidad a través de las TIC es el proyecto E-Culturas, proyecto desarrollado por el Grupo IDEO, de la Universidad de Jaén, y que tenía como objetivo fundamental el establecer una plataforma virtual que sirviera especialmente al alumnado de educación primaria para mejorar su capacidad de intercambio y comunicación cultural con alumnado perteneciente a centros de diversos países latinoamericanos. Apuntando en esta misma dirección, tampoco podemos olvidar los programas educativos de la Unión Europea que promueven el intercambio intercultural y la alfabetización digital. De hecho, tal y como plantea Martínez (2010), el objetivo fundamental de estos programas formativos es reforzar la cohesión social, el desarrollo personal, el diálogo intercultural y la participación activa de los jóvenes ciudadanos europeos. Una de las iniciativas más conocidas es el eTwinning, que permite la comunicación e intercambio de experiencias educativas interculturales entre escuelas distantes geográfica y culturalmente.

Por otro lado, también mencionamos otras experiencias de educación inclusiva e intercultural a través de las TIC, en este caso, en la Comunidad Foral de Navarra, donde en los últimos años se está promoviendo el desarrollo de la interculturalidad a través de las TIC. En este sentido, el Departamento de Educación del Gobierno de Navarra ha convocado resoluciones de financiación o de subvención a proyectos educativos y experiencias de Educación Intercultural, desarrollados en centros de enseñanza, públicos y concertados, de la Comunidad Foral de Navarra. Los proyectos son impulsados por equipos de trabajo formados por profesorado de los centros, y donde pueden colaborar ONG, servicios sociales, ayuntamientos, otras entidades y miembros de comunidades. Algunos ejemplos de los 17 proyectos desarrollados durante el curso 2010/2011, son los siguientes: Diferentes pero iquales en derechos del IES de Berriozar. Llevado a cabo en todos los niveles y área, participa el colectivo socia Entreculturas, y los objetivos fundamentales son: fomentar las relaciones y la empatía en el alumnado; conocimiento de las diferentes culturas y religiones e implicación especial de los delegados de clase en la organización de actividades de educación intercultural. Éstas conducen a la reflexión y la concienciación a través de relatos, juegos, vídeos y jornadas interculturales La evaluación se efectuará mediante encuestas, entrevistas personales a una muestra del alumnado y del profesorado implicado. También nos encontramos con el proyecto titulado Corresponsales sin fronteras del CEIP Te- resa Bertrán de Lis, de Caderita. En este caso, se trata de un proyecto a realizar a través de los medios de información y comunicación (TIC) para el desarrollo de habilidades sociales e interculturales. Estas se introducen por medio de cuentos y juegos del mundo. En la etapa de educación Infantil se desarrollan cuentos y juegos del mundo, así como la elaboración de una sección del periódico escolar y actividades en torno a la televisión, mientras que en Educación Primaria se llevan a cabo actividades tales como murales, programas de radio y T.V., relatos de cuentos del mundo, juegos de otras culturas o exposiciones. Todo ello a partir de una plataforma virtual donde existen numerosos recursos didácticos a disposición de la comunidad educativa. Precisamente, en la Web de Aula Intercultural, del sindicato FETE-UGT, se encuentra todo un apartado dedicado a experiencias dedicadas a la promoción de la educación intercultural a través de las TIC.

Ciertamente, son ya varios los centros educativos que vienen desarrollando proyectos educativos específicos para impulsar y generar interculturalidad a través de las redes sociales y plataformas virtuales en dominios libres. A modo de ejemplo, podemos referirnos al esfuerzo realizado desde los grupos de trabajo organizados en los IES Victoria Kent y Dunas de las Chapas en la provincia de Málaga durante los últimos cursos escolares 2009/2010 y 2010/2011. Probablemente existan experiencias de corte similar a las que podríamos hacer referencia. Sin embargo, la escasa difusión de muchas de las iniciativas llevadas a cabo en los centros educativos limita sus posibilidades de desarrollo en otros contextos. Y aunque los dispositivos digitales podrían introducir importantes mejoras en la divulgación de experiencias exitosas, nos volvemos a encontrar con una clara infrautilización de su potencial para conectar a alumnos, centros y profesores, pero también para servir de soporte a un proyecto intercultural de naturaleza más global.

$\mathrm{Al}$ margen de lo que resta por hacer, debemos tener claro que no se trata sólo de un proceso de transformación tecnológico o de incorporación acrítica de un nuevo espacio de encuentro cultural, sino que el hecho de participar de un proceso de toma de decisiones contribuye a la mejor formación en valores democráticos tanto para el alumnado como para el profesorado, y, por supuesto, para las familias y el resto de agentes de la comunidad educativa. Hablamos, en definitiva, de vivir la escuela y la interculturalidad como una construcción comunitaria participativa (Puigvert y Otros, 2006). Teniendo esto presente, lo que ahora exponemos son algunos de los objetivos más relevantes en relación al aprovechamiento de las TIC y el desarrollo de estrategias prácticas que, desde los principios de la interculturalidad, favorezcan una mayor implicación de la comunidad educativa en los procesos educativos:

- Potenciar la comunicación con la comunidad educativa y su entorno social: hojas informativas, páginas Web, correo electrónico, en el que se divulgue el Centro y su oferta educativa, relación con las AMPAs, e intercambio de información con las familias.

- Fomentar actividades de participación virtual de toda la comunidad educativa, a través de la puesta en práctica de un periódico digital, blogs por cursos o niveles educativos, exposición de webquest por parte de los docentes, foros y debates a través de la plataforma virtual del centro escolar, planteando encuestas, jornadas virtuales de puertas abiertas, etc. 
- Incrementar la utilización de las TIC por parte de toda la comunidad educativa, especialmente de las familias, con el fin de promover su participación activa en la elaboración de los documentos pedagógicos del centro, potenciando la wiki y el foro, así como el correo electrónico como medio de aportar recomendaciones y aportaciones comunitarias a la vida educativa del centro escolar, así como para contactar con el profesorado.

Hemos depositado una gran confianza en la institución escolar y en sus profesionales, pero a todas luces es obvio que las acciones puestas en marcha alcanzarán más y mejores resultados si contamos el apoyo y el compromiso de la comunidad educativa en su conjunto. La gestión colectiva de un desafío que afecta a la sociedad civil en su conjunto posibilitará la puesta en práctica de los principios de una ciudadanía democrática donde, como es bien sabido, la participación y la asunción compartida de las responsabilidades ocupan un lugar central.

\section{Conclusiones finales}

Los numerosos cambios que se han producido en un breve periodo de tiempo y, sobre todo, la velocidad con la que han tenido lugar dichas transformaciones, han sido el origen de una preocupación creciente en torno a la capacidad de la escuela para formar a la ciudadanía del siglo XXI. Y aunque resulta harto complicado establecer prioridades cuando abordamos un asunto de relevancia incalculable como es la educación de los jóvenes, uno de los retos más importantes de nuestro tiempo tiene que ver con el desarrollo de aquellas competencias que, desde el respeto y valoración de una sociedad positivamente multicultural e interconectada, facilitan tanto el conocimiento de otras perspectivas y percepciones sobre la realidad como el análisis crítico de las propias. Ciertamente, sería una falacia negar que en los últimos años se han producido numerosos avances, pero no es menos cierto que en el seno de nuestras escuelas persisten problemáticas de las que ya hemos dado cuenta en otras ocasiones, caso de la necesidad de intensificar las relaciones entre familias, escuela y comunidad, asunto ampliamente demostrado que no precisa que añadamos más o mejores argumentos. Pero en lo que sí hemos de insistir es en la búsqueda de nuevas fórmulas susceptibles de optimizar el nivel de implicación y compromiso de instancias y agentes educadores. Precisamente por ello creemos que una de las claves se sitúa en la mayor rentabilización del espacio digital de comunicación para fortalecer una conectividad de corte intercultural mediante la configuración de nuevos espacios de colaboración y aprendizaje que favorecen el desarrollo de estructuras de trabajo cooperativo en la red. Esto supondrá mejoras en el conocimiento, la comprensión y la convivencia intercultural en los más jóvenes, consumidores y también creadores de significados culturales cada vez más diversos, cambiantes y dinámicos.

Ahora bien, antes de terminar quizás debamos preguntarnos si la buena voluntad de los docentes va a ser suficiente para aprovechar las ventajas que pueden introducir los soportes digitales en los procesos de enseñanza-aprendizaje, o bien si otra de las prioridades se sitúa en el ajuste de la preparación del profesorado a los requerimientos de la sociedad actual, entre ellos, la mejor capacitación para gestionar de manera eficaz las aulas con apreciable heterogeneidad étnico-cultural. Asimismo, el desarrollo de las competencias interculturales en la sociedad del conoci- miento implica que los docentes deben dinamizar propuestas que construyan comunidades de aprendizaje, luego la formación y la comunicación debe ir adquiriendo un matiz comunitario básico en la configuración de redes educativas dinámicas, donde familias, profesorado y alumnado tengan mejores y mayores oportunidades de conocimiento y comprensión intercultural.

\section{Bibliografía}

Abdallah-Pretceille, Martine (2001). La educación intercultural. Barcelona: Idea Books.

Aguaded, Julio I.; Pérez Rodríguez, María A.. (2007). La educación en medios de comunicación como contexto educativo en un mundo globalizador. En J. Cabero (Coord.). Nuevas tecnologías aplicadas a la educación. Madrid: McGraw-Hill, pp.63-75.

Area, Manuel (2010). Enseñar la competencia digital e informacional [diapositiva]. Peñaranda de Bracamonte, 27. http://www.slideshare.net/citafgsr/manuel-areacita2010?from=share_email_logout2. Fecha de consulta: 17.08.2011.

(2009). Las redes sociales en internet como espacios para la formación del profesorado. Razón y palabra, 63. http://www.razonypalabra.org.mx. Fecha de consulta: 5.10. 2010.

Barkley, ElisabethF.; Cross, K.Patricia; Mayor, Claire H. (2007). Técnicas de aprendizaje colaborativo. Madrid: Morata.

Bolívar, Antonio (2008). El discurso de las competencias en España: educación básica y educación superior. REDU. Revista de Docencia Universitaria, 6(2). http://www.redu.m.es/Red_U/m2. Fecha de consulta: 13.09.2012.

Borrero, R. y Yuste, R. (2011). Digiculturalidad.com. Interculturalidad y TIC unidas en el desarrollo del enfoque competencial del curriculum, en Leiva, J. y Borrero, R. (Coords.). Interculturalidad y escuela. Perspectivas pedagógicas en la construcción comunitaria de la escuela intercultural, Barcelona: Octaedro, pp145-164.

Cabero, Julio, López, Eloy; Llorente, María del C. (2009). La docencia universitaria y las tecnologías Web 2.0 Renovación e innovación en el Espacio Europeo. Sevilla: Mergablum.

Coll, C., Bustos, A. y Engel, A. (2007). Comunidades de práctica complementarias: el equipo del programa y la comunidad en línea del curso. Revista Electrónica de la Educación: Educación y Cultura en la Sociedad de la Información, 8(3). http://www.usal.es/ teoriaeducacion. Fecha de consulta: 20.10.2012.

Cummins, Jim; Sayers, Dennis (1995). Brave new schools: Challenging cultural illiteracy through global learning networks. New York: St. Martins Press

De Haro, Juan José (2010). Redes Sociales para la educación. Madrid: Anaya.

De Miguel, Mario (2005). Cambio de paradigma metodológico en la educación superior, Cuadernos de Integración Europea, 2, 6-27.

http://www.cuadernosie.info. Fecha de consulta: 2.06.2011

De Pablos, Juan (Coord.) (2009). Tecnología Educativa. Málaga: Aljibe

Díaz-Aguado, $\mathrm{M}^{\mathrm{a}}$. José (2003). Educación intercultural y aprendizaje cooperativo. Madrid: Pirámide. 
Esteve, José Manuel (2004). La formación del profesorado para una educación intercultural, Bordón, Revista de Orientación Pedagógica, 56 (1), pp.95-116.

Francisco, Andrea (2010). Construyendo ciudadanía participativa en la sociedad de la información. Pixel Bit, 37, 135-146.

http://www.sav.us.es/pixelbit/pixelbit/articulos/n37/ 11.pdf

Kozma, R.B., y Anderson, R.E. (2002). Qualitative case studies of innovative pedagogical practices using ICT. Journal of Computer Assisted Learning, 18, pp.387394.

Jordán, J.A. (1996). Propuestas de educación intercultural para profesores. Barcelona: CEAC.

Leiva, Juan J.; Yuste, R.; Borrero, R. (2011). La interculturalidad a través de las TIC: Caminando hacia la 'digiculturalidad' con las comunidades virtuales de aprendizaje. En A. Hernández y S. Olmos (Coords.) Metodologías de aprendizaje colaborativo a través de las tecnologías (253-263), Salamanca: EUS.

Lluch, Javier; Salinas, Jesús (1997). Del proyecto educativo al aula. 21 ideas para ponerse en marcha, Cuadernos de Pedagogía, 264, pp.54-60.

López, E. (2009). Nuevos escenarios virtuales docentes e innovadores en el marco europeo: Edublog de un profesor universitario. Ponencia presentada al Congreso Internacional Virtual de Educación. CIVE 2009. http://www.steiformacio.com/cive/programa.asp?idioma=2. Fecha de consulta: 21.09.2012.

Luchtenberg, S. (1995). Intercultural Communicative Competence: a challenge in multicultural and antiracist education, European Journal for Intercultural Studies, 6 (2), pp.12-23.

Martín, Mo . Teresa; Quiroz, Catalina (2006). Perfil y funciones del educador social, en López-Barajas, E. (Coord.). Estrategias de Formación en el Siglo XXI. Barcelona: Ariel, pp. 285-316.

Martínez, Francisco (2010). Las redes digitales como marco para la multiculturalidad. Alcalá de Guadaíra (Sevilla): MAD.

Martínez, Francisco; Prendes, M.Paz (2003). ¿A dónde va la educación en un mundo de tecnologías?, en Martínez, F. (Comp.). Redes de comunicación en la enseñanza. Las nuevas perspectivas del trabajo corporativo. Barcelona: Paidós; 281-300.

Merino, David; Ruiz, Crsitóbal (2005). Actitudes de los profesores hacia la educación intercultural, Aula Abierta, 86, 185-204.

Merino, David; Leiva, Juan; Pedrero, E. (2012). La educación intercultural como propuesta de innovación educativa en una escuela inclusiva. Actas del XV Congreso Nacional y V Iberoamericano de Pedagogía. Burgos: Universidad de Burgos.

Merino, José V. (2009). La escuela centrada en la comunidad. Un modelo de escuela inclusiva para el siglo XXI. Revista Complutense de Educación, 20, pp.33-52. http://revistas.ucm.es/index.php/RCED/index

Mominó, Josep M.; Sigalés, Carles; Meneses, Julio (2007). La escuela en la sociedad red. Internet en la educación Primaria y Secundaria. Barcelona: Ariel.

Monereo, Carles (2009). Internet, un espacio para desarrollar las competencias básicas, en Monereo, C. (Coord.), Internet y competencias básicas. Aprender a colaborar, a comunicarse, a participar, a aprender. Barcelona: Graó; 5-26.

OCDE (2005). The definition and selection of key competencies. Executive Summary.

http://www.oecd.org/dataoecd/47/61/35070367.pdf. Fecha de consulta: 28.08.2012

Parrilla, Angeles (2002). Acerca del origen y sentido de la educación inclusiva. Revista de Educación, 327, pp.1129.

Prendes, M. Paz.; Castañeda, Linda; Gutiérrez, Isabel (2010). Competencias para el uso de TIC de los futuros maestros. Comunicar, 35, 175-182.

Prenksy, Marc (2001). Digital Natives, Digital Immigrants: Do they really think differently? On the Horizon, 9(6), $1-6$

http://www.marcprensky.com/writing/prensky\%2o\%20digital\%2onatives,\%20digital\%20immigrants\%20-\%2opart2.pdf. Fecha de consulta: 26.09.2012.

Priegue, Diana; Leiva, Juan (2012). Las competencias interculturales en la sociedad del conocimiento: reflexiones y análisis pedagógico. EDUTEC, Revista

Electrónica de Tecnología Educativa, 40.

http://edutec.rediris.es/Revelec2/Revelec4o/compete ncias_interculturales_sociedad_conocimiento_reflexiones_analisis_pedagogico.html. Fecha de consulta: 05.10.2012.

Puigvert, L. et al. (2006). Teorías y Sociedades Dialógicas. Nuevas transferencias ciencia y sociedad en la era del conocimiento. Madrid: Ministerio de Ciencia y Tecnología.

Pujolás, Pere (2010). No es inclusión todo lo que se dice que lo es. Aula de innovación educativa, 191, pp.38-41. http://www.fapac.cat/system/files/aula_1.pdf

Rogoff, Barbara (1993). Aprendices del pensamiento. El desarrollo cognitivo en el contexto social. Barcelona: Paidós.

Santos, Miguel; A.; Lorenzo, Ma . Mar (2009). A educación para a cidadanía e os profesores. Visión e desafio. Vigo: Xerais.

Santos, Miguel A.; Lorenzo, Ma ${ }^{\mathrm{a}}$. Mar; Priegue, Diana (2009). Aprendizaje cooperativo: práctica pedagógica para el desarrollo escolar y cultural. Magis, Revista Internacional de Investigación en Educación, 1(2), pp.289-304.

http://revistas.javeriana.edu.co/index.php/MAGIS/ar ticle/view/3385

(2011). (Red). Conectando a los profesores para el desarrollo de la interculturalidad. Revista Educación $X X 1$ (en prensa).

Slavin, R.E. (1999). Aprendizaje cooperativo. Teoría, investigación y práctica. Buenos Aires: Aique.

Thomas, John W. (2000). A review of research on project-based learning. San Rafael, CA: Autodesk Foundation.

Tondeur, Jo et al. (2009). Understanding structural and cultural school characteristics in relation to educational change: the case of ICT integration, Educational Studies, 35 (2), 223-235.

Tubela, Inma y Vilaseca Jordi (2005). Sociedad del conocimiento, cómo cambia el mundo ante nuestros ojos. Barcelona: UOC.

Valls, R. et al. (2002). Comunidades de aprendizaje. Transformar la educación. Barcelona: Graó. 
Vilà, Ruth (2005). La Competencia Comunicativa Intercultural. Un estudio en el primer ciclo de la ESO. Barcelona: Universidad de Barcelona. Tesis Doctoral.

Vinagre, Margarita (2010). Teoría y práctica del aprendizaje colaborativo asistido por ordenador. Madrid: Síntesis. 